\title{
Mathematical modeling of a MOSFET transistor as modulator in AM transmission
}

\section{Modelado Matemático de un transistor MOSFET como modulador en transmisión en AM}

MOTA-GALVÁN, Eduardo†*\& REYES-MARTINEZ, Roberto Alejandro

Universidad Autónoma de Baja California, Faculty of Chemical Sciences and Engineering

ID $1^{\text {st }}$ Author: Eduardo, Mota-Galván / ORC ID: 0000-0003-3380-6759

ID $1^{\text {st }}$ Co-author: Roberto Alejandro, Reyes-Martinez / ORC ID: 0000-0003-2210-2692, Researcher ID Thomson: AAN2895-2020, CVU CONACYT ID: 21470

\begin{abstract}
The necessary methodology is presented to characterize the alternating signal transistor in the time and frequency domain and obtain its characteristic equations including its transfer function. The behavior of the transistor gate is studied in different models of manufacturers in alternating signal, therefore the difference between the behavior relationship between the theory and the information obtained in the experimentation is shown. All of the above to have an optimization, control or description of the operation of a real transistor and be used in an electrical / electronic application in general, in this case, for an AM modulation.
\end{abstract}

MOSFET, Mathematical modeling,
Modulated amplitude

\section{Resumen}

Se presenta la metodología necesaria para caracterizar el transistor en señal alterna en el dominio temporal y frecuencial y obtener su(s) ecuaciones características incluyendo la función de transferencia del mismo. Se estudia el comportamiento de la compuerta del transistor en diferentes modelos de fabricantes en señal alterna por tanto se muestra la diferencia entre la relación de comportamiento entre lo teoría y la información obtenida en la experimentación. Todo lo anterior para tener una optimización, control o descripción del funcionamiento de un transistor real y ser aprovechado en una aplicación eléctrica/electrónica en general, para este caso, para una modulación AM. MOSFET, Modelado matemático, Amplitud
modulada

\footnotetext{
* Correspondence to Author (e-mail: eduardo.mota@uabc.edu.mx)

$\uparrow$ Researcher contributing as first author.
} 


\section{Introduction}

The amplitude modulation is the process to modify the amplitude of a carrier signal, usually high frequency sinusoidal, commonly in RF spectrum, proportionally to the value of amplitude of a low frequency modulator signal [1]. Compared to other forms of modulation, AM modulation is easier, with the disadvantage of low quality signal. To modulate in AM it is necessary to use no lineal devices or signal multipliers.

\section{There are two types of AM:}

- $\quad$ DSBFC (double side band full carrier) is the easier method and common to make it. It is called double side band because, in the frequency domain, the bandwidth of the modulating signal is on both sides of carrier signal. Therefore the bandwidth of transmission is two times the frequency of modulating signal causing a loss in the bandwidth in the spectrum.

SSB (single side band) in this type of modulation, it is only transmitted one of both side band of information, eliminating the carrier signal and the excess side band. The disadvantages are the bad quality sound compared with DSBFC AM, as well as the signal reception is more complex.

A lot of source of information either bibliography or digital, they explain the theoretical form of Am using established mathematical equations depending of the transmitter circuit. This transmitter usually is built with BJT transistor or JFET transistor. This transistors usually appear in the bibliography with a schematic diagram with some equation explaining the behavior of the transmitter.

However, the information is limited about the AM using MOSFET transistors at least in the traditional form of amplitude modulation without explaining of the behavior, matheatical equation or any transference function that allows to know the model.

From this some questions arise for modulators with BJTs or JFETs:
Where does the equation for the behavior of the transistor come from? What physicalmathematical criteria did they use to obtain it? Why don't they usually use AM modulators with MOSFETs if it is a power device with better efficiency, compared for example with a BJT?

It is important to know the origins and criteria to develop the equation of transmitter and this form take advantage the experience and knowledge to modeling other transistors or devices. If it is possible also apply the above in bibliography or exercises in laboratory practices. The central hypothesis of this investigation is the possibility to make an amplitude modulation taking advantage the no lineal behavior of MOSFET Gate transistor consider the transistor is stable to high frequency.

With the data from measurements it is possible to find behavior equations in time and frequency domain and lay the groundwork to the characterization of any transistor or device.

In this article talks about of the form of characterize a channel $\mathrm{N}$ MOSFET transistor, for that is presented the section of methodology, test prototype implementation, results obtained, conclusions and future work.

\section{Metodología}

The process of obtain data as well as the develop of characterization/modeling is recommended take in consideration the flow diagram which is presented in fig. 1

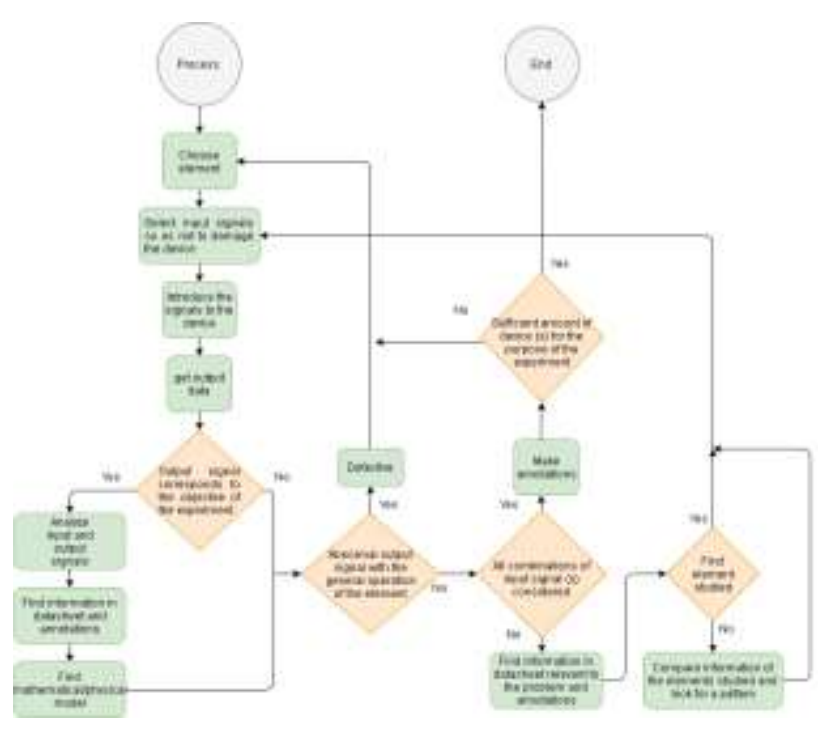

Figure 1 Process to obtain data Source: Own Elaboration 
In the figure 1, it shows the methodology to make test measurements of any element or device, to know if the element is defective or if the quantity of test elements are enough to the investigation. To find the appropriate mathematical or physical model, the methodology used is presented in the figure 2 .

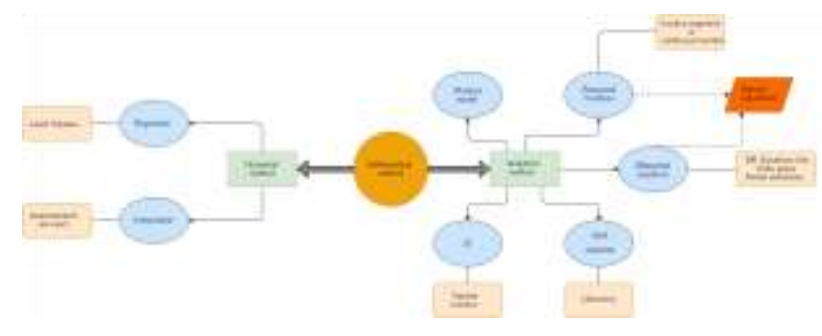

Figura 2 Process to select a mathematical model Source: Own Elaboration

\subsection{Modeling (data fit)}

When an experiment is developing, it is necessary capture input and output values to understand the behavior of the experiment or the element to obtain an appropriate model acceptable; considering the method is not exact due approximations in the experiment or errors in the measurements for example inherent, truncation or rounding. For this is considered to use common methods for example regression or approximation method, interpolation method and analytic method which will give a brief explanation.

\subsection{Regression or approximation method}

By definition this method is a relationship that allows predict the probably values of one variable from another variable. This method is used when is desired a function that approximate the data obtained due errors in measurements or in devices. The order of the function is minor than quantity of points obtained in the measurements. There are distinct types of regression: lineal, quadratic, logaritmic, exponential, polynomial and least squares [2][3].

\subsection{Interpolation Method}

By definition the interpolation method is the estimation of a value of the variable $y$ for a certain value of the variable $\mathrm{x}$. This method is used when is desired a function to connect each of the data obtained, assuming that the measurements or the devices are exact and there is no error. The maximum power of an interpolation polynomial function is equal to the number of points minus one [4].

\subsection{Analytic method}

This method is the most "exact" because only use mathematical functions such as polynomials, algebraic or trigonometric functions that approximate the data, without use directly the data obtained from the experiment, it means, it is used only as a comparison graph of the results with the proposed model. The selection of an analytical method depends on the characteristics of the element or experiment [5].

Once identified the possible methods to proceed to modeling, it is necessary to identify an element of study, which for the interest of this investigation turns out to be the MOSFET transistor that is briefly described.

\section{MOSFET transistor}

The MOSFET transistor is a transistor whose operation is different from a BJT transistor. In a BJT transistor, the current that flows through it is controlled by an input current, it means, it is a current device controlled by current. However the MOSFET transistor is a transistor where the current flow from pin $\mathrm{D}$ to pin $\mathrm{S}$ being controlled by voltage across pin $\mathrm{G}$.

Where the 'D' connector represents the drain which is where the current enters and the ' $\mathrm{S}$ ' connector is the source where the current leaves. The main structure of a MOSFET transistor is a metal oxide semiconductor capacitor that corresponds to the gate $(\mathrm{G})$, this capacitor is isolated from the semiconductor substrate that represents the drain and the source.

The semiconductor substrate can be made of material $\mathrm{N}$ or material $\mathrm{P}$, depending on the type of material the voltage $\mathrm{V}$ can be negative or positive. For example, in the case of a P-type substrate, it is necessary for the voltage to be positive at the $G$ pin so that the few free electrons found in the substrate are attracted by the electric field generated. In this form, the electrons will produce an "enrichment" segment in the substrate, creating an N-type channel through which the input current to the transistor can pass. If the substrate is made of $\mathrm{N}$ material, a P-type channel will be created. The enrichment channel is volumetric, that is, it has a length and width defined by the manufacturer [6]. 
Starting with the use of an N-channel MOSFET transistor, will be made a model from a modulation circuit.

\section{Implementation}

To test a MOSFET transistor in modulation mode there are 3 forms of connection which is presented in the figures 3,4 and 5.

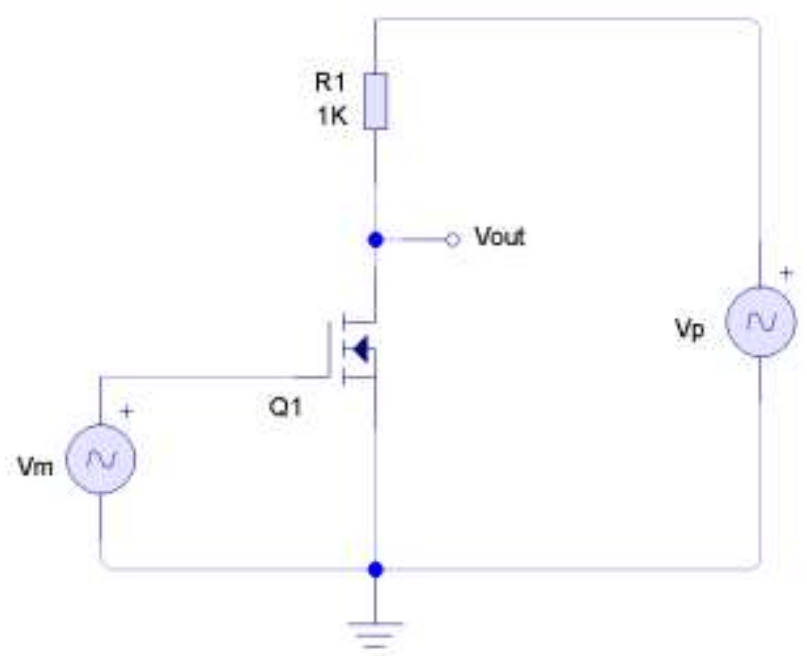

Figure 3 Connection 1, carrier signal to Drain and modulating singal to Gate

Source: PCB Wizard

In figure 3 the carrier signal is applied to the drain of the transistor, while the modulating or information signal is applied to gate, in this form take advantage of the no linear regions of the transistor. The output signal $\mathrm{V}_{\text {out }}$ is the carrier modulated by the signal coming from the gate.

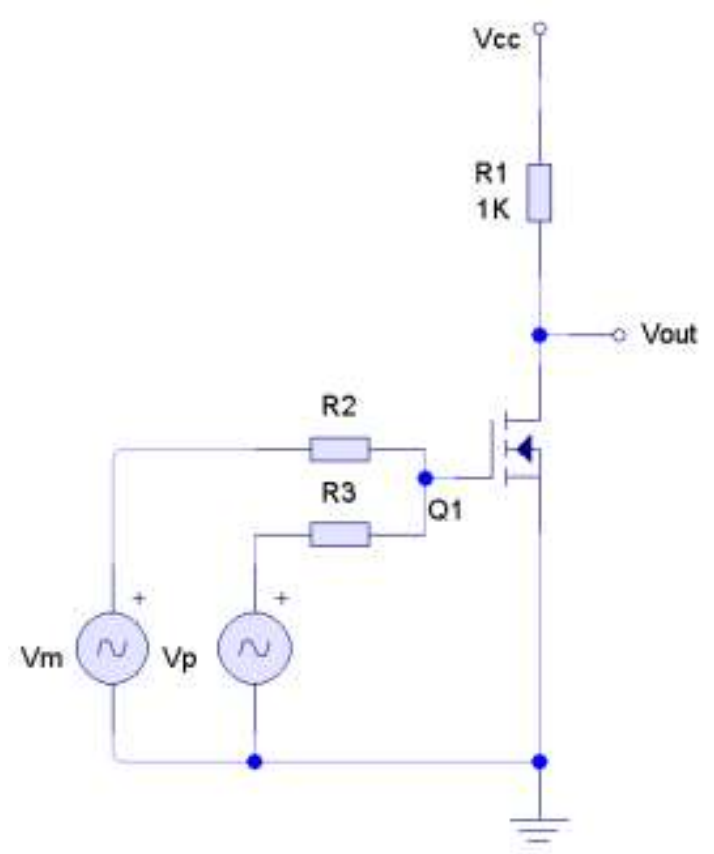

Figure 4 Connection 2, carrier and modulating signals to Gate, $V_{D C}$ to Drain

Source: PCB Wizard.
In the figure 4 an arrangement is presented where is applied a direct voltage $\mathrm{V}_{\mathrm{CC}}$ to the drain of the transistor, however in the Gate is applied the sum of the carrier signal and the modulating signal, obtaining in Vout a signal that consist the harmonics of the sum of these signals.

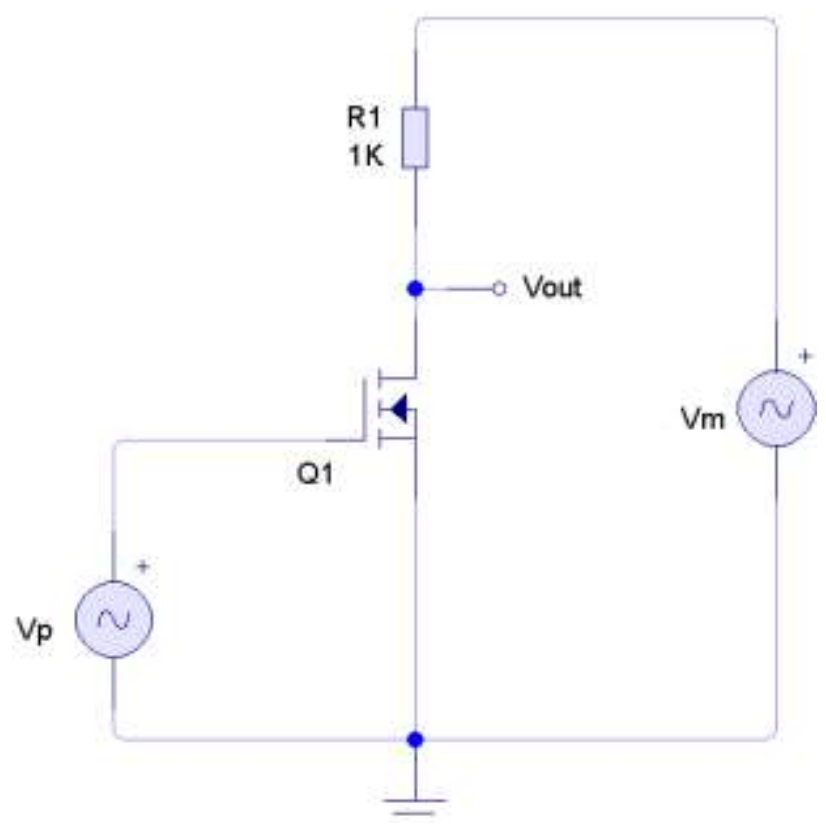

Figure 5 Connection 3, Carrier signal to Gate and modulating signal to Drain.

Source: PCB Wizard.

In the figure 5 the connections of the modulator circuit are similar to figure 3 with the difference in connection change of the signals in the transistor, for this case, the modulating signal is applied to Drain of the transistor and the carrier signal is applied to Gate.

The amplitude of the modulated signal in the circuit of figure 3 is greater than that obtained by the circuit of figure 5 . In the arrangements of figures 4 and 5 the best results are obtained with the difference that in figure 4 use more elements.

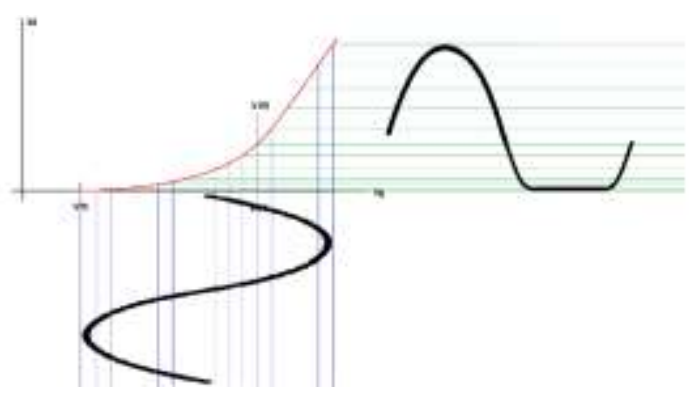

Figure 6 Drain current as a function of transistor gate voltage

Source: Own Elaboration 
In the figure 6 shows the ideal behavior of a MOSFET transistor, the curve that represent a quadratic expression (similar to an exponential) represents the drain current Id as a function of the gate voltage $\mathrm{Vg}$.

A MOSFET transistor has 3 types of operation:

\subsection{Cutting region}

In this mode of operation happens when the voltage Vgs is less than Vth, this means that the enrichment channel is not formed, therefore there is no current from drain to source.

\subsection{Saturation or active region}

This mode of operation happens when the voltage Vgs is between Vth and VDS and this region is purely quadratic, this is due the enrichment channel is formed but not completely, that means, when Vgs increases, the channel will form according to the field electrical increase.

\subsection{Lineal or ohmic region}

It happens when VDS is greater than Vgs, this region is linear or ohmic because the channel is completely formed and the linear zone ascends with a positive slope. Where $\mathrm{Kn}$ is a parameter given by the manufacturer, which involves the dimensions of the channel as well as the electrical characteristics [6]. According to what is established in previous paragraphs, we can summarize the behavior of a MOSFET transistor as follows:

$$
I_{d}=\left\{\begin{array}{c}
I_{d 0} e^{\frac{V_{g}-V_{t h}}{n V_{t}}} V_{t h}>V_{g} \\
K_{n}\left[2\left(V_{g}-V_{t h}\right) V_{d s}-V_{d s}^{2}\right] V_{t h}<V_{g}<V_{d s} \\
K_{n}\left(V_{g}-V_{t h}\right)^{2} V_{g}>V_{d s}
\end{array}\right.
$$

With the help of figure 6 , it is possible to have an idea of how the drain current behaves according to the connections of figures 4,5 and 6 . To use many transistors and make the tests that allows to obtain a model, was developed a fixture which is a $4 \times 4 \mathrm{~cm}$ PCB designed in the programs Livewire and PCB Wizard, it is possible to polarize and add the input signal, as well as to measure the signals of interest and the substitution of the transistor for the different tests, this fixture is shown in figure 7.

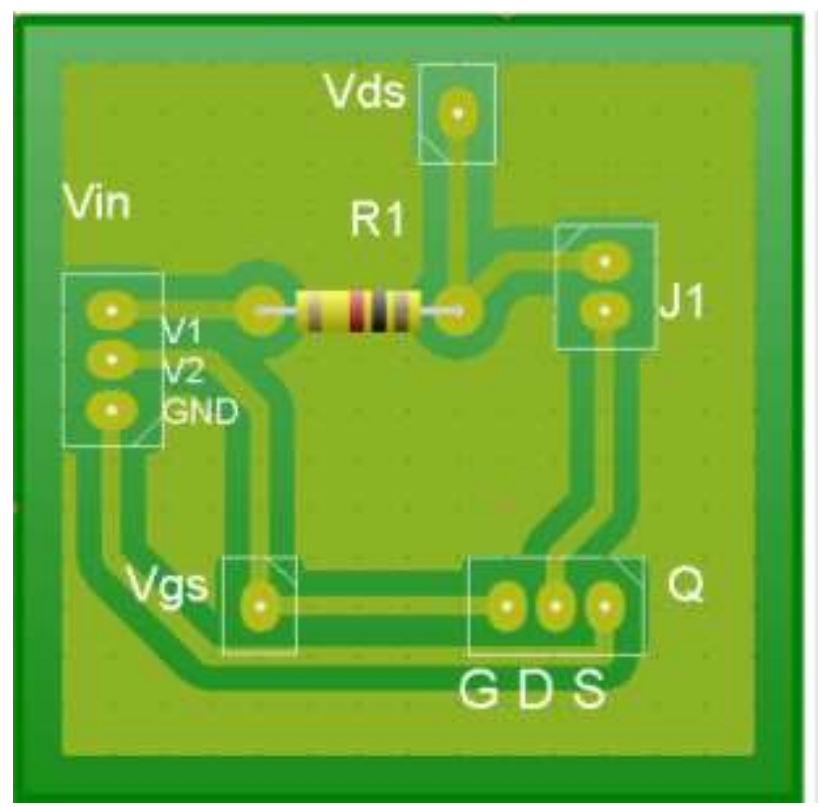

Figure 7 PCB design

Source: PCB Wizard.

The connection points Vin and Q are by means of 3-terminal headers, where $\mathrm{Q}$ relates to the terminals for the test transistor and Vin the bias. Vds, Vgs and $\mathrm{J} 1$ are male header connectors. The Vgs and Vds connectors are used to make measurements easily, while $\mathrm{J} 1$ is a jumper that is used to measure the current flowing through the drain of the transistor.

\section{Results}

Once the fixture was implemented, the circuit was polarized using the method of connecting (figure 4), with which measurements were made to the transistors described in table 1. These Measurements, previously, were made with variations in $\mathrm{Vg}$ from $0 \mathrm{~V}$ to $5 \mathrm{~V}$ with increments of $0.01 \mathrm{~V}$ tabulating and graphing the results.

\begin{tabular}{|l|l|l|l|}
\hline \multicolumn{1}{|c|}{ Parameters } & \multicolumn{1}{c|}{ Qgs } & \multicolumn{1}{c|}{ Ciss } & \multicolumn{1}{c|}{ Vth } \\
\hline RFP2N08 & - & $200 \mathrm{pF}$ & $2 \mathrm{a} 4 \mathrm{~V}$ \\
\hline IRF9Z10 & $3.8 \mathrm{nC}$ & $270 \mathrm{pF}$ & $-2 \mathrm{a}-4 \mathrm{~V}$ \\
\hline 2N60B & $2.2 \mathrm{nC}$ & $380-490 \mathrm{pF}$ & $2 \mathrm{a} 4 \mathrm{~V}$ \\
\hline IRL3303 & $8.8 \mathrm{nC}$ & $870 \mathrm{pF}$ & $1 \mathrm{~V}$ min $^{*}$ \\
\hline IRF640N & $11 \mathrm{nC}$ & $1160 \mathrm{pF}$ & $2 \mathrm{a} 4 \mathrm{~V}$ \\
\hline IRF640C & $13 \mathrm{nC}$ & $1300 \mathrm{pF}$ & $2 \mathrm{a} 4 \mathrm{~V}$ \\
\hline K3878 & $34 \mathrm{nC}$ & $2200 \mathrm{pF}$ & $2 \mathrm{a} 4 \mathrm{~V}$ \\
\hline
\end{tabular}

Table 1 Comparative of analyzed transistors Source: Own acquisition. $* V_{d s}=V_{g s}, I_{d}=250 \mu \mathrm{A}$

As an example of the measurements carried out, the following cases are presented as representative of the work: 


\subsection{Measurements of parameters using K3878 TOSHIBA transistor}

The experiment was made with a circuit adding the K3878 MOSFET increasing the gate-tosource voltage by $0.01 \mathrm{~V}$ starting from $0 \mathrm{~V}$ and ending at 5V. The circuit was polarized with $\mathrm{Vcc}$ $=5 \mathrm{~V}$ in direct and values were taken in the parameters Vgs, Ids, Vds and leading to the graphical interpretations that are presented in graph 1.
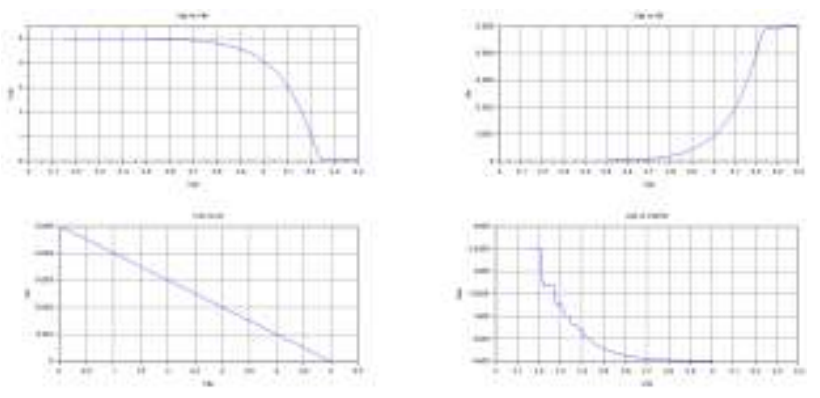

Graph 1 transistor parameters from left to right: $\mathrm{V}_{\mathrm{gs}} \mathrm{vs}$ $\mathrm{V}_{\mathrm{ds}}, \mathrm{V}_{\mathrm{gs}}$ vs $\mathrm{I}_{\mathrm{ds}}, \mathrm{V}_{\mathrm{ds}}$ vs $\mathrm{I}_{\mathrm{ds}}, \mathrm{V}_{\mathrm{gs}} \mathrm{vs}\left(\mathrm{V}_{\mathrm{ds}} / \mathrm{I}_{\mathrm{ds}}\right)$

Source: Scilab.

In the graph 1 shows the input and output characteristics of K3878 MOSFET transistor, for example transfer function of voltage (top left graph), transfer function of current (top right graph), output resistance (lower left graph) and transfer function of output resistance (lower right graph).

\subsection{Measurements of parameters using IRF640N transistor}

Applying the same procedure of data collection but substituting the MOSFET K3878 by IRF640N, the results is obtained that is presented in the graph 2.
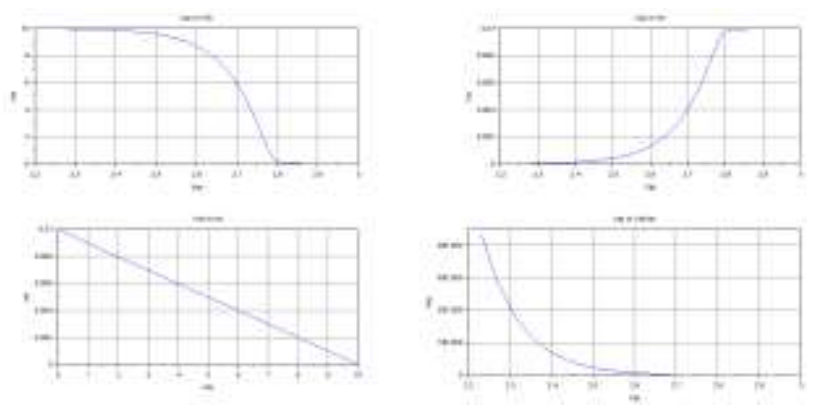

Graph 2 transistor parameters from left to right: $\mathrm{V}_{\mathrm{gs}} \mathrm{vs}$ $\mathrm{V}_{\mathrm{ds}}, \mathrm{V}_{\mathrm{gs}}$ vs $\mathrm{I}_{\mathrm{ds}}, \mathrm{V}_{\mathrm{ds}}$ vs $\mathrm{I}_{\mathrm{ds}}, \mathrm{V}_{\mathrm{gs}}$ vs $\left(\mathrm{V}_{\mathrm{ds}} / \mathrm{I}_{\mathrm{ds}}\right)$ Source: Scilab
In the graph 2 shows the input and output characteristics of IRF640N MOSFET transistor, for example transfer function of voltage (top left graph), transfer function of current (top right graph), output resistance (lower left graph) and transfer function of output resistance (lower right graph). Comparing the graphs 1 and 2 it can observe that the voltage threshold in both transistors is different, as well as is indicated in the table 1 and both transistors reach to lineal region long before of $\mathrm{V}_{\mathrm{ds}}$.

\subsection{Comparison of measurements with theory}

To make the comparison between the literature indicates with the obtained data from measurements, its necessary take as reference the measurements obtained from K3878 TOSHIBA as shown in the graph 3.

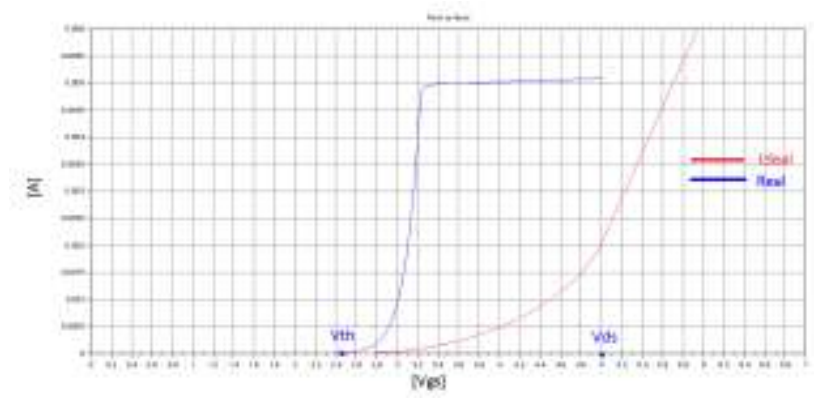

Graph 3 Comparison of transistor K3878 with an ideal behavior.

Source: Scilab

In the graph 3 the exponential line that is to the right of the graph represents the behavior of an ideal MOSFET transistor according to the literature and represented in equation (1). This behavior indicates that there is a quadratic no linear region when Vgs is between Vth and Vds. When Vgs is greater than Vds, the transistor channel is formed proportionally to Vgs, that is why the corresponding exponential growth, however, when data is captured and transferred to a graph, it is identified that the behavior of the transistor does not comply with was expressed in equation (1), giving a representation that is closer to the origin (a lower Vgs).

Observing both behaviors (transistor $\mathrm{K} 378$ and IRF640N) the conclusion is that a real transistor does not behave as the theory explains, also the transistor reaches the ohmic region long before Vds and the no linear behavior is not presented as a Quadratic function, for which the opportunity arises to identify the mathematical models that represent the device, for this, are used some techniques mentioned above.

MOTA-GALVÁN, Eduardo \& REYES-MARTINEZ Roberto Alejandro. Mathematical modeling of a MOSFET transistor as modulator in AM transmission. Journal of Technological Engineering. 2021 


\subsection{Polynomial equations in region of transistor K3878}

Due to what was presented in the behavior of the K3878 transistor, it was proceeded to find the polynomial or exponential equations that could represent its behavior and allow a characterization of the transistor as approximately as possible to its operation, for this, it was necessary to use the trend line of Libre Office spreadsheet.
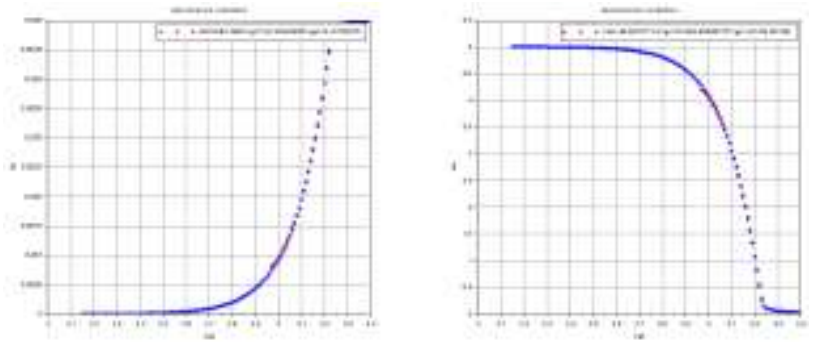

Graph 4 Quadratic approximation $I_{\mathrm{ds}} \mathrm{vs} \mathrm{V}_{\mathrm{gs}}$ (left) y $\mathrm{V}_{\mathrm{ds}} \mathrm{vs}$ $\mathrm{V}_{\mathrm{gs}}$ (right)

Source: Scilab.

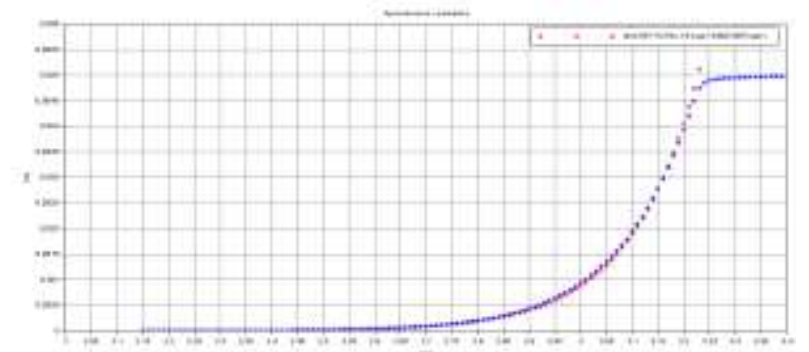

Graph 5 Exponential approximation $\mathrm{I}_{\mathrm{ds}} \mathrm{vs} \mathrm{V}_{\mathrm{gs}}$ Source: Scilab

In the graph 4 it is identified that it is possible to approximate with a quadratic function a section of the behavior of the transistor, however it does not correspond to what is described in equation (1), the theory explains that the quadratic region of a MOSFET transistor is located between $\mathrm{Vth}$ and $\mathrm{Vds}$ and in graph 4 this is out the limits in theory. moreover it is possible to model the same behavior with an exponential function, covering all the no linear region, which is desirable and does not correspond to an ideal behavior.

\subsection{Relation of deformation of output signal as function of frequency of the transistor}

An interesting point observed in the measurements is the signal deformation of the transistor in Vds and Ids, as a function of frequency, identifying that this deformation is directly proportional to the applied frequency in Vgs,
Therefore it is necessary a investigation process of the variables involved that allow to identify the behavior.

For this experiment were used the transistors presented in table 1 . These were tested using a value of

$V g s=2.5 \sin (2 \pi 200000 t)+2.5 V_{d c}$

with a bias $\mathrm{V}_{\mathrm{cc}}=5 \mathrm{~V}$ and using the connection in the figure 4.

For this investigation the results obtained re resented as the best and worst performance, in terms of signal deformation. The best performance was presented by the RFP2N08 transistor as shown in figure 8 , while the worst performance was obtained with the $2 \mathrm{~N} 60 \mathrm{~B}$ transistor as shown in figure 9.

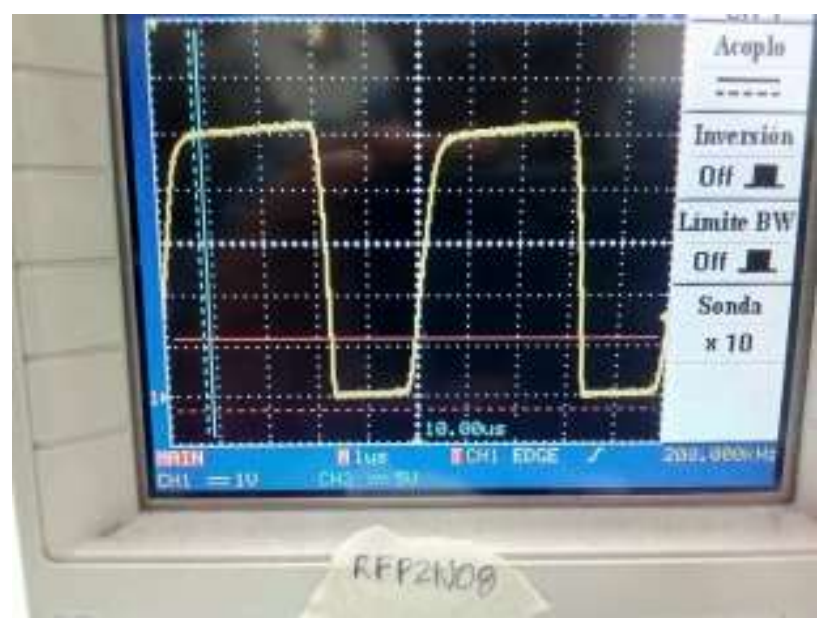

Figure 8 Deformity due to frequency of RFP2N08 transistor

Source: Own acquisition.

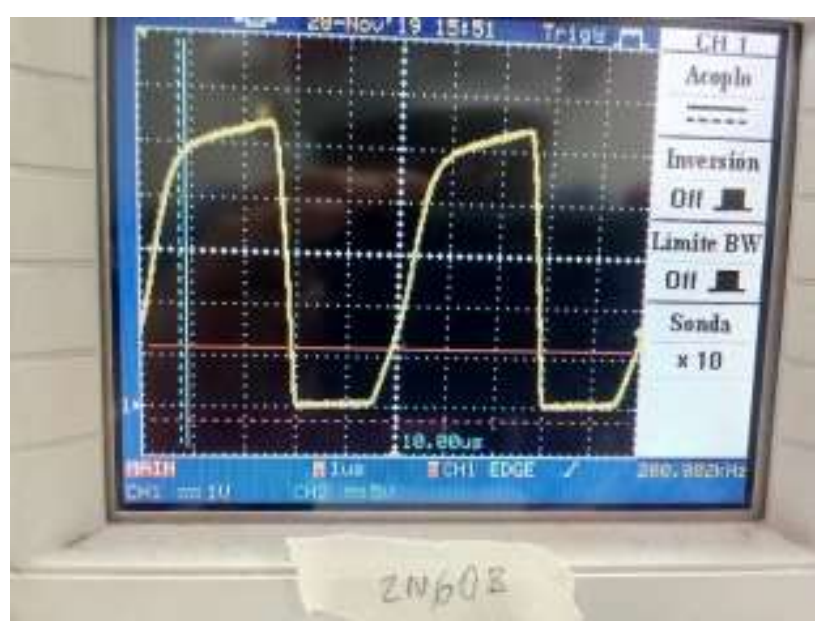

Figure 9 Deformity due to frequency of 2N60B transistor Source: Own acquisition.

MOTA-GALVÁN, Eduardo \& REYES-MARTINEZ, Roberto Alejandro. Mathematical modeling of a MOSFET transistor as modulator in AM transmission. Journal of Technological Engineering. 2021 
The performance of these transistors was compared from best to worst to find which are the variables involved in the deformation of the signal as a function of frequency. Analyzing and reading the datasheets of each of the transistors in Table 1, the variables were found that correspond to Qgs and Ciss.

According to table 1 and the previous measurements, it is concluded that the deformation as a function of the frequency of a MOSFET transistor is inversely proportional to the value of Qgs or Ciss, it means, when more small are these two values, the frequency that the transistor can handle without deforming is greater as shown in graph 6 .

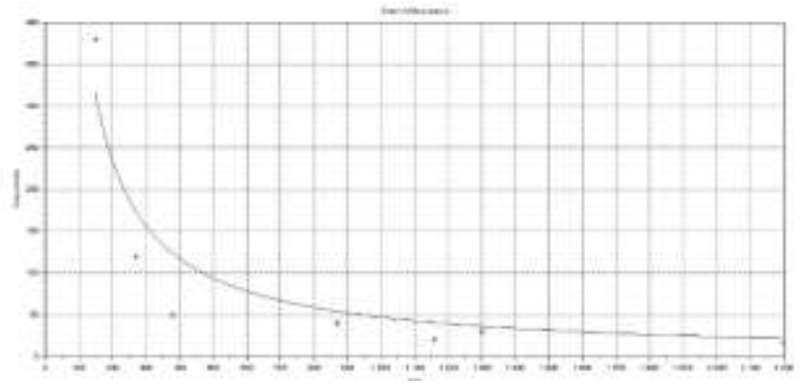

Graph 6 Relation between $C_{\text {iss }}$ and frequency in Gate without deformation

Source: Scilab.

Graph 6 shows the relationship of a MOSFET transistor with the internal capacitance of the channel with respect to the maximum frequency that the gate can handle without deformation, preserving the characteristics of the transistor.

$f_{t}=\frac{a_{0}}{C_{i s s}}$

where $f_{t}$ is the frequency transistor y $a_{0}$ is the coefficient obtained by least square model.

\subsection{Deformation of $V_{d s}$ as function of the frequency}

The behavior of the transistor (RFP2N08) is presented as a case study with the value of Vgs presented in expression (2), making variations in the frequency of the signal with steps of $10 \mathrm{KHz}$ between each test from $25 \mathrm{KHz}(25,35,45,55$, $65 \mathrm{KHz}$ ) highlighting the deformity of the Vds output signal.

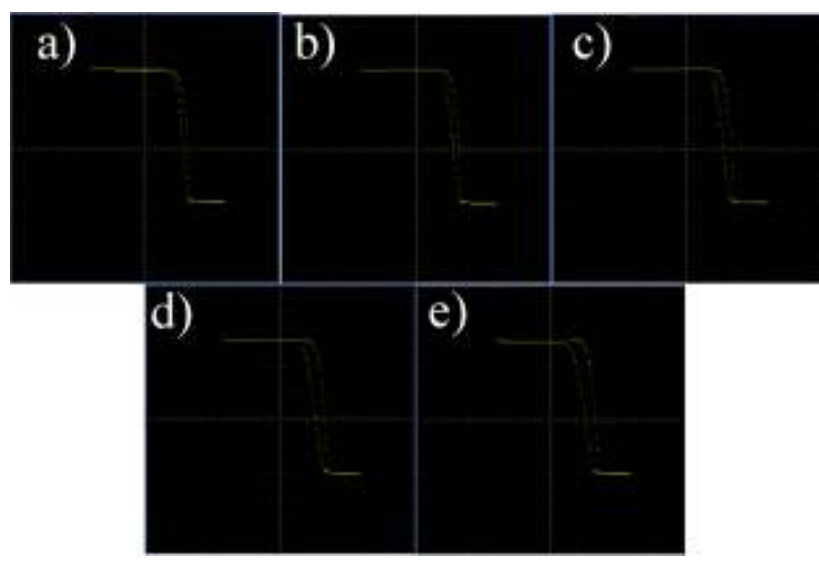

Graph 7 From left to right $\mathrm{V}_{\mathrm{ds}}$ signal with 25, 35, 45, 55 y $65 \mathrm{KHz}$ respectively

Source: Tektronix MDO3024

To proceed with obtaining the transistor model, the signal defined in (2) with a frequency of $45 \mathrm{KHz}$ for the RFP2N08 transistor, obtaining the numerical data and its graph as shown in graph 8.
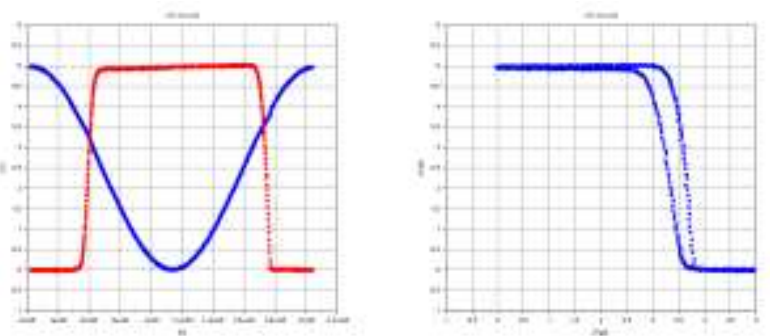

Graph $8 \mathrm{~V}_{\mathrm{ds}}$ signal with $45 \mathrm{KHz}$ using a osciloscope as data acquisition

Source: Tektronix MDO3024.

Different linearizable numerical mathematical models were tested using the least squares method programmed in Scilab, obtaining the following model:

$V_{d s}=\sum_{n=0}^{13} a_{n} v_{g s}^{n}$

Where $a_{n}$ are the coefficient obtained by the last square model which approximate the $\mathrm{V}_{\mathrm{ds}}$ function.

Equation 4 represents the Vds output as the sum of powers, which is valid up to power number 13 . 


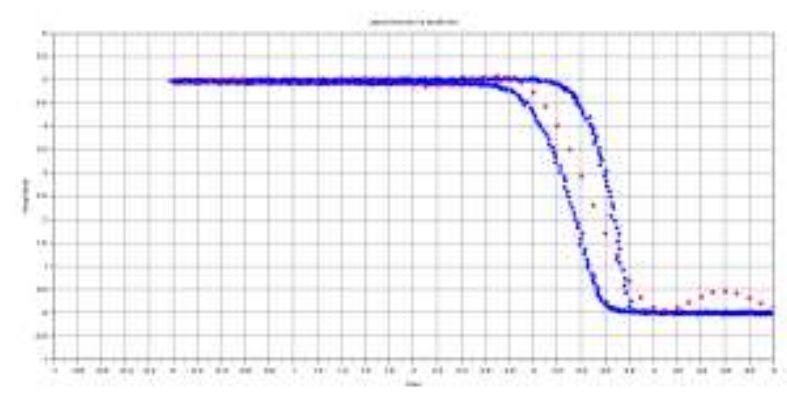

Graph 9 Comparison of the output voltage Vds (blue line) respect to the mathematical model (red dotted line) Source: Scilab.

One of the advantages of the numerical approximation is that it considers a large part of the no linear region of the Vds signal, as well as that it is a more compact mathematical model, however it is not very exact because the model approximates to the no linear region that contains two different values, also, the model is only valid until you do not oscillate. Therefore, it was necessary to find other numerical models, being identified as pertinent by Fourier Series, which the corresponding characteristic polynomial was obtained, remaining as:

$$
\begin{aligned}
& V_{d s}=a_{0}+\sum_{n=1}^{5} a_{n} \cos (2 \pi 45000 n t)+ \\
& a_{n+1} \sin (2 \pi 45000 n t)
\end{aligned}
$$

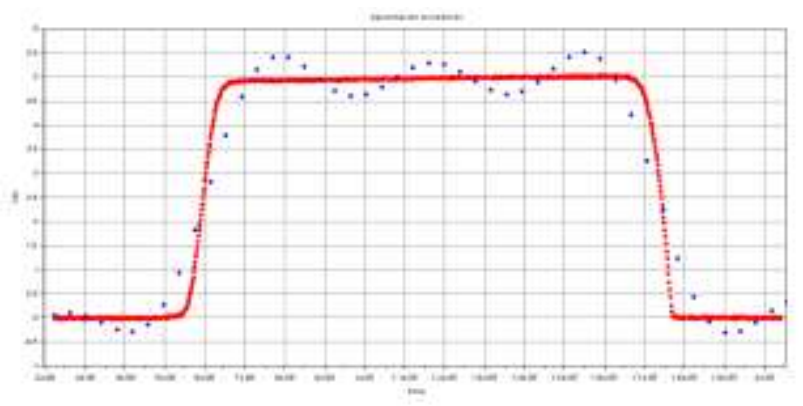

Graph 10 Comparison of the Vds output signal of the model (5) (oscillating line in blue) with the real measurement (almost continuous line in red)

Source: Scilab

\subsection{Frequency domain of transistor harmonics}

For the test of this model, the same procedure was followed that was obtained by least squares, with a carrier signal as the one presented in equation (2), with frequency of $45 \mathrm{KHz}$ and applying the model presented in (5), as well as the analysis with FFT (fast Fourier transform) resulting in what is presented in graph 11 .
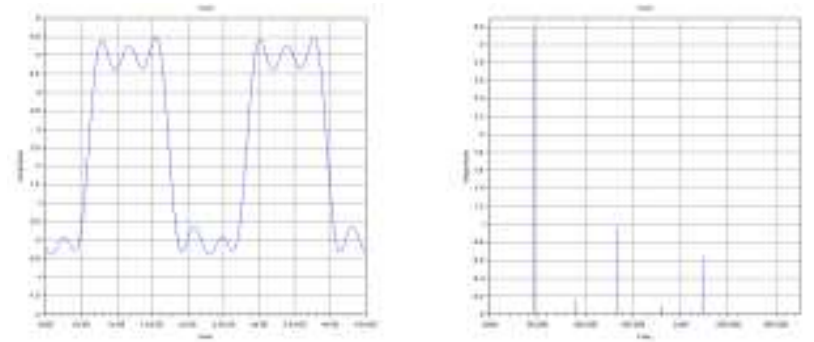

Graph $11 \mathrm{~V}_{\mathrm{ds}}$ modeling and frequency spectrum Source: Scilab.

The model (5) does not approximate the no linear region of the $\mathrm{Vds}$ signal that present in the temporal domain compared with the model (4). In the frequency domain due to the harmonics of model (5) it is a good candidate to be used as an AM modulator because it describes more harmonics than are necessary for modulation. The model (5) is more compact and simple but without many harmonics. Analyzing the model (4) and applying a signal like the one presented in (2) with $45 \mathrm{Khz}$ and applying FFT:
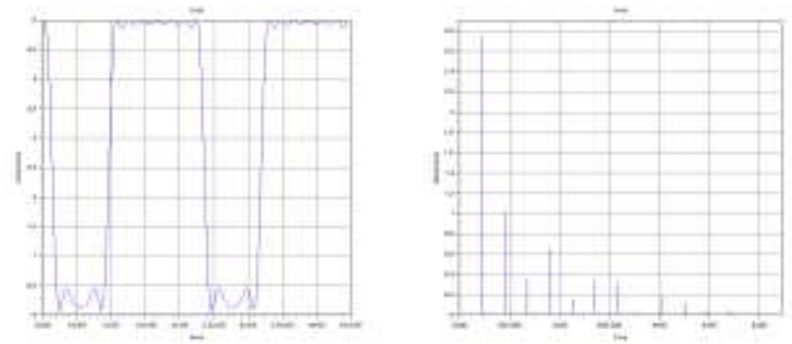

Graph $12 \mathrm{~V}_{\mathrm{ds}}$ modeling and frequency spectrum Source: Scilab.

Comparing graphs 11 and 12 the conclusion found is the model (4) is the only that is approximated to the $\mathrm{Vds}$ signal since it handles the sum of powers and these add more necessary harmonics. In graph 12 there are more harmonics which do not appear in graph 11 .

Another numerical model was proposed for $\mathrm{Vds}$ as a function of $\mathrm{Vgs}$, taking one of the two hysteresis lines of the transistor that is most stable in frequency changes in blue color (Graph 8), modeling only the no linear region and applying an analysis with FFT. The numerical approximation model for the no linear section of $\mathrm{Vds}$ as a function of $\mathrm{Vgs}$ is

$V_{d s}=\sum_{n=0}^{6} a_{n} v_{g s}^{n}$ 


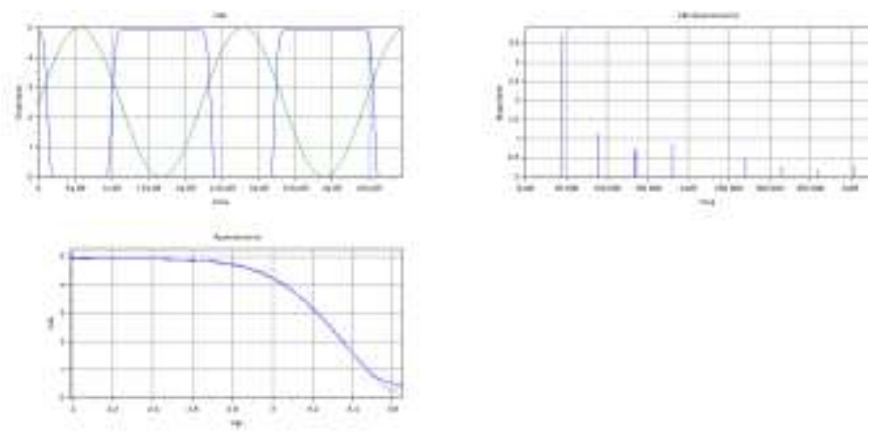

Graph $13 \mathrm{~V}_{\mathrm{ds}}$ modeling and frequency spectrum using only the no linear region

Source: Scilab.

The model (6) presents a greater approximation in the temporal and frequency domain and with less processes compared to model (4) because some harmonics do not appear and they do not approximate with the results of Graph 14.

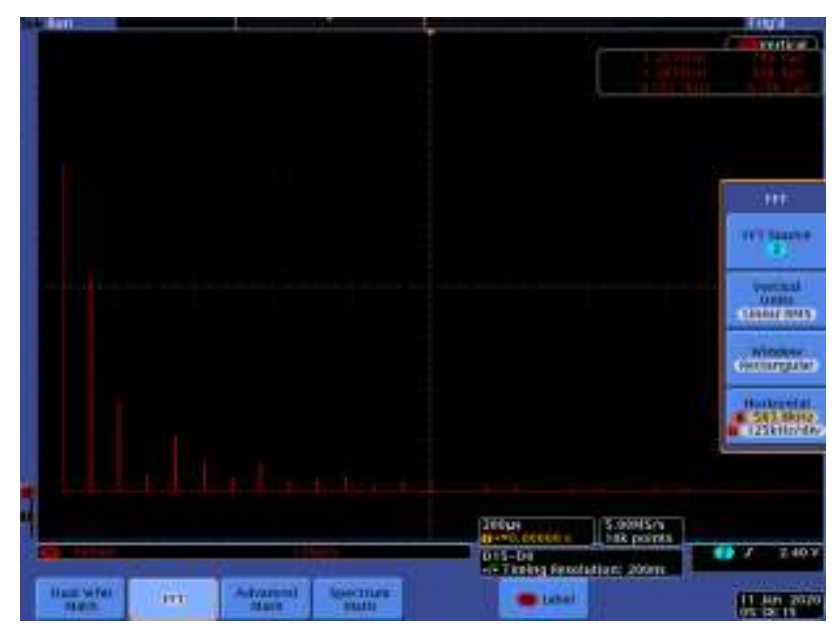

Graph 14 Measured frequency spectrum of the transistor Source: Tektronix MDO3024

\section{Gratitude}

To the Autonomous University of Baja California and especially to the Faculty of Chemical Sciences and Engineering and to my advisor for contributing with the materials and equipment necessary to make the corresponding tests to develop this research.

\section{Conclusions}

The present work has allowed to identify that the traditional model of a MOSFET is not the only model or the most approximated to a real device, that is possible to have different approximations with a greater or lesser degree of approximation and that it is posible to make in voltage and in frequency, depending on the mathematical model as a polynomial algebraic model or a combination of transcendent functions.
Obtaining a suitable mathematical model transistor it is possible to use it for specific applications such as an AM modulator.

\section{References}

[1] Tomasi, W. (2003). Sistemas de comunicaciones electrónicas. Pearson educación.

[2] Sauer, T., \& Murrieta, J. E. M. (2013). Análisis numérico . Pearson Educación.

[3] Chapra, S. C., Canale, R. P., Ruiz, R. S. G., Mercado, V. H. I., Díaz, E. M., \& Benites, G. E. (2011). Métodos numéricos para ingenieros (Vol. 5). McGraw-Hill.

[4] Vidal,M.[Uniersitat Politecnica de Valncia UPV].(2017,octubre,4)Una introducción al ajuste polinómico de datos por mínimos cuadrados, utilizando Matlab || UPV.[Archivo de video].Recuperado de youtube.com/watch?v=d2wGLqWyoKg

[5] Bocco, M. (2010). Funciones elementales para construir modelos matemáticos.

[6] Neamen, D. A. (2007). Microelectronics: circuit analysis and design (Vol. 43). New York: McGraw-Hill.

Gerald, C. F. (1987). Análisis numérico. Representaciones y Servicios de Ingeniería.

Burden, R. L., Faires, J. D., \& Iriarte Balderrama, R. (1996).Análisis numérico. 